\title{
EVALUATION OF AGV ROUTING STRATEGIES USING HIERARCHICAL SIMULATION
}

\author{
Ralf W. Seifert \\ Department of Mechanical Engineering \\ University of Karlsruhe (TH) \\ Karlsruhe, GERMANY
}

Michael G. Kay
James R. Wilson

\author{
Department of Industrial Engineering \\ North Carolina State University \\ Raleigh, NC 27695-7906, U.S.A.
}

\begin{abstract}
To analyze the operation of an arbitrary AGV system under selected vehicle routing strategies, we present a simulation model that can handle multiple system layouts, a varying number of AGVs, and a varying number of pedestrians moving around the system. We introduce a dynamic vehicle routing strategy based on hierarchical simulation that operates as follows: at the time of each routing decision for an $\mathrm{AGV}$ in the main simulation, subsimulations are spawned for each of a varying number of alternative routes; and the performance observed in these subsimulations is then used to make the routing decision in the main simulation. A case study illustrates the advantages of this strategy.
\end{abstract}

\section{INTRODUCTION}

The major material handling link in an automated material handling system is provided by Automated Guided Vehicles (AGVs). As part of a modern material handling system, these AGVs are driverless vchicles following physical or virtual guidepaths under the control of a computer. They play the key role in interconnecting all important locations on the factory floor for the horizontal movement of materials in a flexible manner. Unlike other more conventional material handling devices, an AGV can select its own path among many routes to reach a designated workstation or warehouse. To realize an AGV system's full potential for flexibility, we have to exercise carcful planning and control over the design and operation of the system.

The problem of assigning parts to vehicles and vehicles to parts has been the focus of several studies, and numerous authors have evaluated heuristic dispatching rules such as the Random Vehicle Rule, the Nearest Vehicle Rule, the Longest Idle Vehicle Rule, and the Least
Utilized Vehicle Rule (Viswanadham and Narahari 1992). However, determining the "nearest" vehicle and the "shortest" path itself is not an easy problem. We observe that the shortest travel-distance path may not be the shortest travel-time path. Along a path, the actual travel speed of a vehicle depends on the amount of congestion encountered. This can have significant influence on the travel time and hence on the performance of the system.

\subsection{Problem Statement}

To gain the full benefits from an AGV system, we must exploit the flexibility of the AGVs; in particular, we must take advantage of the possibility to choose between various paths to reach a certain destination. In order to find the path with the shortest travel time, we must solve the well-known vehicle routing problem (Hodgson et al. 1987). Beyond the deterministic approach that simply follows the shortest travel distance, extensive research has been directed towards finding algorithms to calculate the stochastic shortest path in a probabilistic network. However, the complexity of the problem at hand makes it difficult, if not impossible, to obtain effective analytical solutions. While the deterministic approach does not account for any probabilistic influences on the travel time, the stochastic shortest path calculation is in general limited to networks that have mutually independent arc lengths (Andreatta 1987). This is an unacceptable limitation because, for example, the occurrence of pedestrians along the arc currently being traversed by an AGV is dependent on the occurrence of pedestrians along the arcs previously traversed by that AGV. There is a clear need for a more realistic approach to the assignment of the "shortest travel-time path" to a specified destination given the present status of the system. Such an approach would provide further potential for an improvement in the use of AGVs. 


\subsection{Terminology and Problem Representation}

According to Co and Tanchoco (1991), the primary vehicle management functions for AGVs can be defined as follows:

- Dispatching is the process of selecting and assigning tasks to vehicles.

- Routing is the selection of the specific paths taken by vehicles to reach their destinations.

- Scheduling is the determination of the arrival and departure times of vehicles at each segment along their prescribed paths to ensure collision-free journeys.

The focus of this study is to explore the benefits of dynamic vehicle routing strategies versus conventional static strategies for controlling the operation of the AGV system.

The guidepaths of an AGV system can be modeled as a graph consisting of nodes connected by a set of arcs. The nodes represent key locations in the AGV system such as intersections and Pickup or Delivery (P/D) stations. The arcs connecting these nodes represent segments of the physical or virtual guidepaths to be followed by an AGV. This graph is the primary input to the routing function of the AGV control system. Given the location of an AGV and its prescribed destination, the vehicle router can find the sequence of nodes that specify the path of the vehicle. Alternative routes can be evaluated based on an aggregate cost of traversing a sequence of arcs, such as travel distance or travel time.

The evaluation of alternative routes and the actual routing of a vehicle based on this evaluation can be either static or dynamic:

- When routing is static, the path taken by an AGV between any two given nodes is fixed. The simplest solution is always to select the path with the shortest travel distance.

- When routing is dynamic, different paths can be taken by an AGV at different times in moving between two given nodes. Taking into consideration the current status of the system, the vehicle router selects a path for the AGV at the time when the vehicle is dispatched (Hodgson et al. 1987); and, if there is a communications link between the router and the vehicle, then the router modifies the vehicle's path during travel.

\subsection{Global Vision As Information Support}

To achieve the most efficient path selection, we must be able to route vehicles dynamically over uncongested routes or to avoid obstacles in the way of an AGV. This basically means we need to have not only a more sophisticated control scheme for the AGVs but also a system feedback mechanism. Both tasks may be realized by using free-ranging AGVs under the control of a global vision system. The global vision system enables the control system to detect congestion or unexpected obstacles and thus provides the necessary feedback for globally efficient path planning.

Global vision refers to the use of cameras (or other types of sensors) placed at fixed locations in a workspace to extend the local sensing available on board each vehicle in a free-ranging AGV system. Information from the cameras is used to $(a)$ monitor the workspace to detect and track potential obstacles both in the immediate vicinity of each AGV and over its intended path; $(b)$ track each AGV along its intended path to bound errors in the vehicle's dead-reckoning sensors; (c) monitor the load aboard each AGV to detect positioning errors; and (d) provide video images of the entire workspace so that a human operator can monitor the status of operations throughout the facility. The status of the AGV system is available as input to high-level transport control functions (Kay 1992).

Given that this system information can be provided in the present study, we seek to evaluate the use of a computer simulation model as a short-term decision tool to account for the current system status and to determine the current optimal path with the minimum travel time to reach a certain destination. This cannot be accomplished by the routing strategies that do not utilize a system feedback mechanism.

\section{MODEL DESCRIPTION}

\subsection{Overview of the Model}

The simulation model developed in this research (Seifert 1994) is a discrete-event, mixed-language model written in SLAM II (Pritsker 1995) with all event-processing routines written in the $\mathrm{C}$ programming language (Kernighan and Ritchie 1988). The model has a flexible design and can handle multiple user-specified layouts for AGV systems. Complete control of the layout and the parameters of the AGV system are provided to fulfill the requirements of an effective design tool. By collecting various statistics, the software provides a complete bottleneck analysis at the end of each simulation experiment. Pedestrians and the other AGVs are the two possible causes of congestion for each vehicle. While requiring a minimum of user input, the software allows the user to:

1. Specify a varying number of AGVs and pedestrians in the system as well as different speeds for loaded and unloaded vehicles;

2. Set node-specific parameters, such as the time delay at intersections caused by the need for vehicles to 
slow down in order to detect and avoid possible collisions; and

3. Study the effect on the system throughput of the loading/unloading times at specific P/D stations.

Furthermore, the model is capable of evaluating the potential improvement in the utilization of an AGV system that can be achieved by an increase in routing flexibility. We introduce, implement, and evaluate a new dynamic vehicle routing strategy that is based on hicrarchical simulation and that operates as follows: at the time of each routing decision for an AGV in the main simulation, we spawn subsimulations for cach of a varying number of alternative routes; and the observed performance of the system in these subsimulations is then used to make the routing decision in the main simulation.

\subsection{Detailed Model Description}

\subsubsection{General Information Structure}

Using the distance matrix of a layout provided as user input in a plain text (ASCII) file, we can specify the existence and the physical length of each arc connecting two nodes in the network. This information is then stored in SLAM II files called layout files, with a unique layout file corresponding to each node (Pritsker 1974. pp. 244-246). The first entity in each layout file represents the node itself. The following entities in a layout file represent the branches emanating from this node. The attributes of the entities in the layout files provide the information used to create path information and to model the operation of the AGV system. In the course of the simulation, the status of the system is reflected by the changing values of the attributes of these entities.

The AGVs and pedestrians are represented by entities that are placed on the event calendar and are processed at the corresponding event times. An AGV or pedestrian entity arriving at the end of a directed branch or turning into the next branch as a part of its current route accesses the attributes of the node entity and the branch entities defining the current conditions at that location in the system. Depending on the attribute values for the arriving AGV or pedestrian, the current local conditions are then interpreted and the disposition of the arrival is determined.

\subsubsection{Stochastic Model Components}

To improve the accuracy of the results of each simulation experiment, the model has been carefully constructed to take advantage of the variance reduction technique of common random numbers (Law and Kelton
1991, pp. 613-628). This means that in each vehicle routing scenario to be compared, the same pattern of demands (destinations) is generated for the same AGV (while different demand patterns are generated for different AGVs); and a similar approach is used to generate destination patterns for pedestrians. Thus we obtained an efficient approach for studying the effect of varying the total number of AGVs and pedestrians in the system, and we enhanced the comparison of different routing and sensing strategies. However, the functions used to generate and return the next demand (destination) for each $\mathrm{AGV}$ and pedestrian have a modular design so that these functions may be easily replaced or modified to read the next demand from an external scheduler.

The self-congestion of AGVs is modeled by using SLAM II files for the intermediate storage of an AGV whenever immediate processing of that AGV is not feasible. If one AGV is blocked, then other AGVs may be delayed as well.

To model the interactions between the AGVs and the pedestrians in the system, we specified first the logic regulating the movement of pedestrians in terms of the following assumptions:

1. Routing of pedestrians is deterministic-that is, given a (randomly generated) destination, each pedestrian will follow the shortest travel-distance path to that destination.

2. Pedestrians do not overtake an AGV traveling on a branch unless the AGV is blocked.

3. If pedestrians are blocked at a node currently being traversed by an AGV, then when the node is made available again, all waiting pedestrians are allowed to cross this node at once prior to any AGV that is also waiting at the same node.

4. Pedestrians do not interact with an AGV currently undergoing a load or unload process at a node; thus a stationary AGV does not influence the movement of a pedestrian.

5. Pedestrians do not interact with each other-that is, they do not cause self-congestion.

6. After a pedestrian reaches its current destination node, the pedestrian will stay at this location for an exponentially distributed holding time before being assigned a new destination and starting to walk to that destination.

A pedestrian traversing a node will block all AGVs attempting to arrive at that node. Further interaction between pedestrians and AGVs is caused by a pedestrian walking on a particular branch since a minimum safety time-distance separation must be maintained for any subsequent AGV traveling on the same branch. 


\subsubsection{Model Verification and Validation}

In addition to the standard trace reports available in SLAM II, we developed several special output reports to provide a means for:

1. Verifying the usage of path information in the course of the simulation experiment;

2. Keeping track of the movements and the blockage of the AGVs and the pedestrians; and

3. Tracing how many subsimulations were spawned at each decision epoch and which routing alternatives were finally selected.

Customized trace reports provide the means for verifying the performance and the validity of the simulation model. In the course of the simulation experiment, the movement of each individual AGV and each individual pedestrian is traced and the corresponding event-times are listed. To verify the implemented logic for modeling the self-congestion of the AGVs and the interaction of the AGVs with the pedestrians present in the system, we provided the appropriate status messages to trace the disposition of the entities in the system as they are processed at their discrete-event times. Furthermore, we listed the current values of each entity and the current travel route that each entity follows.

\section{VEHICLE ROUTING STRATEGIES}

In this section, we detail the static and dynamic vehicle routing strategies implemented in the simulation model.

\subsection{Static Deterministic Vehicle Routing Based on Shortest Travel Distance}

In the deterministic vehicle routing strategy, it is assumed that the best route for a vehicle to take always involves the shortest travel distance. This routing strategy identifies the shortest travel-distance path between each pair of nodes in the layout; and for a given pair of origin and destination nodes, the corresponding identified path is followed at all times regardless of the degree of congestion along the different routes connecting the given nodes. This deterministic vehicle routing strategy is a common approach, and the results obtained by this method may serve as the basis for the evaluation of different routing strategies. However, the deterministic approach reflects the lowest level of routing strategy sophistication in the sense that the vehicle router does not dynamically account for any feedback that is provided on the current status of system congestion. The deterministic approach ignores the likelihood of congestion, obstacles, and breakdowns on the different path segments during travel of the AGVs.

\subsection{Dynamic Stochastic Vehicle Routing Based on Hicrarchical Simulation}

Static deterministic vehicle routing as described in the previous subsection provides very little flexibility. The lack of responsiveness to the changing environment makes the AGV system prone to traffic congestion and blockage caused by system disturbances. To investigate the potential for using the current status of the system for the initialization of one or more short-term subsimulations, we developed a general concept in which subsimulations can be embedded at each decision epoch to mimic the future operation of the system. The observed performance of the system for each of the alternative routes is then passed back to the main simulation and serves as the basis for the current vehicle routing decision. This approach enables the user to make better routing decisions, because it is based on the current status of the system.

During the course of the simulation of the AGV system (referred to as the main simulation), we spawn an appropriate number of subsimulations to support the dynamic vehicle routing decision for an AGV (taking the current status of the AGV system into consideration) whenever the AGV requests a routing decision for traveling to the next assigned destination. The main simulation thereby reflects the operation of the real AGV system, while each subsimulation evaluates a possible routing alternative for the AGV by tentatively advancing the simulation time beyond the present epoch. Thus the key components of the main simulation and the subsimulation model are identical; however, the initialization of the subsimulation relies on the information about the current status of the AGV system as provided by the main simulation. To pass this information to the subsimulation, we store the current status of the main simulation in unformatted files; and then we read these files to initialize the subsimulation. The subsimulation is performed up to the point at which the current AGV reaches its next assigned destination by traveling on the currently specified route. After the subsimulation terminates, the travel time to the assigned destination on the specified route is passed back to the main simulation; and this information provides a basis for the actual routing of the AGV in the main simulation.

The number of alternatives that are evaluated by separate subsimulations depends on the results observed in those subsimulations. First, the AGV is routed on the shortest travel-distance path; and this routing decision is then evaluated by comparing $(a)$ the travel time to the assigned destination that is observed in the subsimulation, and $(b)$ the theoretically smallest possible time to travel on an alternative route as determined by the physical length of the alternative route. If the travel 
time $(a)$ observed on the shortest travel-distance path is less than the minimum travel time $(b)$ on the next best alternative route, then we do not need to spawn another subsimulation to support the routing decision for the current AGV. Consequently we route the AGV on the shortest travel-distance path in the course of the main simulation, since the AGV would be routed on the shortest travel distance path in the real AGV system.

However, if an alternative route promises to yield a reduced travel time for the current $\mathrm{AGV}$, then we cvaluate this alternative route by a separate subsimulation. After this subsimulation terminates, we compare the results obtained from all of the subsimulations performed so far to the theoretical minimum travel times for the remaining alternative routes. By this means, we determine whether we already have obtained sufficient information to select the most promising alternative route for minimizing the travel time of the current $\mathrm{AGV}$. If this is the case, then we send the AGV along the route that required the least travel time for the AGV to reach its destination; and we proceed by restarting the main simulation. Otherwise, we continue to evaluate additional alternate routing decisions for the current AGV if they are promising, and we again apply the same comparison after each subsimulation. Using this approach to the generation of subsimulations, we try to minimize the total number of subsimulations performed to support the final routing decision.

The main simulation terminates at the time specified in the control input file for the overall simulation experiment.

\section{CASE STUdy}

In this section, we briefly present a case study of an AGV system; and we perform formal statistical comparisons for the evaluation of the different vehicle routing strategies with a varying number of AGVs and a varying number of pedestrians in the system. Figure 1 depicts the layout of the AGV systcm used in the case study. This system contains 10 pickup/delivery stations that are numbered 1 to 10 .

\subsection{Formulation of the Main Performance Measure}

To evaluate the performance of the AGV system, we formulated a specific performance measure referred to as the "relative delay" of an AGV. The statistics collection on the relative delay of an AGV is performed when an AGV reaches its assigned destination. To calculate the relative delay of an AGV, we compute the difference between $(a)$ the AGV's actual travel time to its current destination, and $(b)$ the corresponding theoretical minimum travel time of the AGV as determined by its speed and the physical length of the shortest-travel-distance path between the AGV's current origin and destination nodes. The relative delay of an AGV is used to compare the differences in system performance that are caused by applying different vehicle routing strategies, since it is our objective to minimize the travel time between all pairs of nodes in the network. In addition, given the total simulated time of the operation of the AGV system, the total number of observations of the relative delay for all AGVs (which reflects the total number of completed assignments) is used to calculate the throughput per AGV per hour for the current system configuration and vehicle routing strategy.

It is obvious that the realized throughput of the AGV system is directly related to the observed relative delay of the AGVs in the system. The relative delay experienced by an AGV goes up as we increase the congestion by increasing the total number of AGVs in the system; and as the relative delay of an AGV increases, the throughput realized by the $\mathrm{AGV}$ system decreases. If we increase the level of system disturbance modeled as pedestrians walking in the system and causing dependent branch disturbances, then we can furthermore see that an increased level of system disturbance leads to an increase in the relative delay of the AGVs and a decrease in the throughput achieved by the system.

\subsection{Experimental Comparison of Routing Strategies}

To evaluate the performance of the different vehicle routing strategies implemented in the simulation model for a varying number of AGVs and a varying number of pedestrians in the system, we performed a formal statistical analysis of the results using the method of common random numbers. Figures 2 and 3 depict $90 \%$ confidence intervals for the reduction in the expected relative delay of AGVs that is achieved by using the dynamic stochastic vehicle routing strategy based on hierarchical simulation when this strategy is compared to the static deterministic strategy based on the shortest traveldistance path. The results graphed in Figures 2 and 3 are based on 10 independent pairs of replications each of length 480 minutes, where each pair of replications correspond to the routing strategies that are being compared.

We sce from Figures 2 and 3 that as the number of pedestrians in the system increases, the superiority of the hierarchical simulation approach becomes more pronounced. Moreover, the average reduction in the relative delay of AGVs appears to be significant even if the level of system disturbance is fairly low. However, it is not clear that these conclusions can be extrapolated to other AGV systems with different layouts. Notice that in Figures 2 and 3, we also plot the reduction in relative 


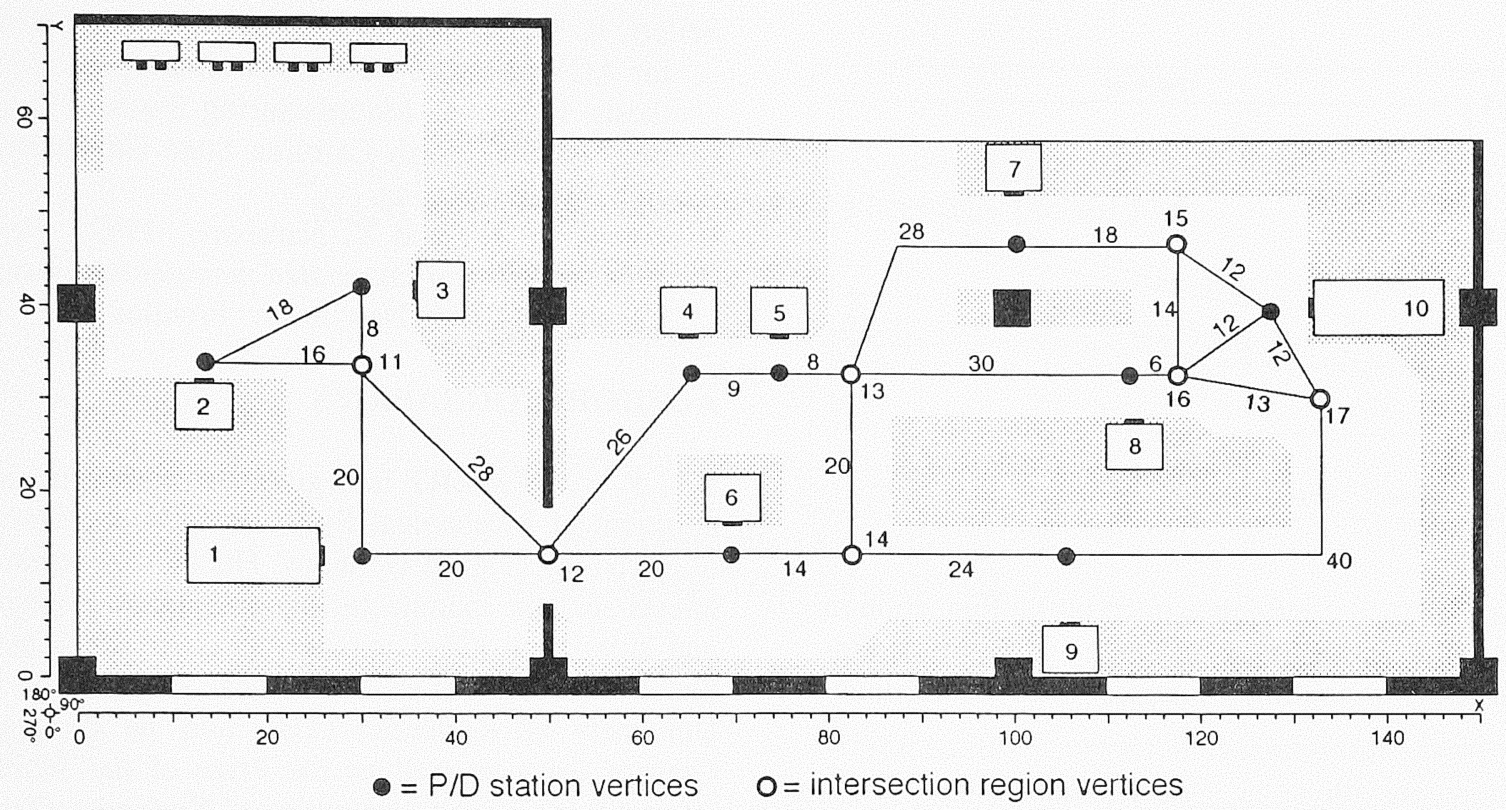

Figure 1: Layout of the AGV System for the Case Study

delay achieved by a static stochastic vehicle routing strategy based on iterative simulation-generated estimates of the stochastic shortest path; for complete details on this strategy, see Seifert (1994).

\section{CONCLUSIONS AND RECOMMENDATIONS}

In this research, we demonstrated the potential advantages of dynamic AGV routing strategies based on hierarchical simulation. The results of the case study indicated the superiority of this approach in comparison to the usual static vehicle routing strategy based on the deterministic shortest travel-distance path. However, these results cannot be generalized without much more extensive experimentation. Moreover, to enjoy the full potential benefits of the dynamic vehicle routing approach, we have to account for the capabilities of this approach during the design phase of the AGV system by including more flexibility in AGV system design. Specifically, the AGV system design should (a) provide a sufficient number of alternative paths so that critical bottlenecks can be bypassed dynamically, $(b)$ allow for dynamic selection of the stations corresponding to functionally equivalent workcenters, and $(c)$ allow for varying degrees of sensing capabilities to provide information concerning the congestion status of the system, ranging from only local, vehicle-based sensing to full global vision capabilities.

Enhancements to our method for exchanging information between the main simulation and the subsimulations should enable the user to implement dynamic vehicle routing strategies in a real-time decision support tool for controlling the operation of an AGV system. In particular, the use of shared memory may eliminate much of the input/output overhead required by our approach to hierarchical simulation of AGV systems. Finally, this approach naturally lends itself to implementation on parallel processors.

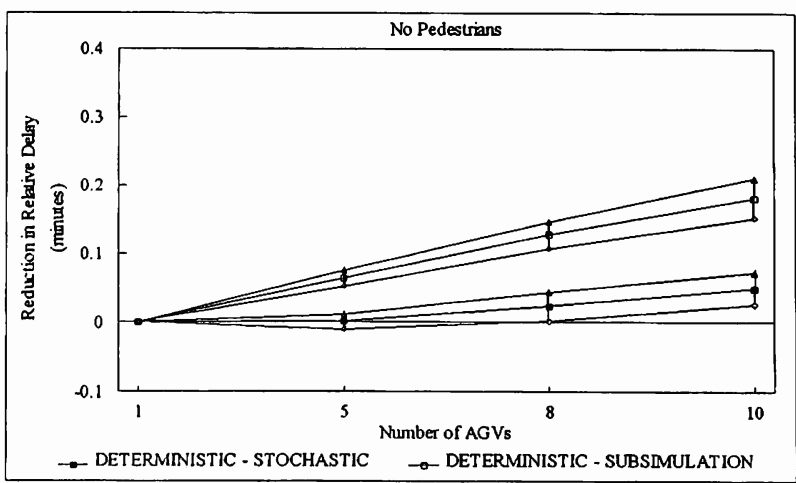

Figure 2: $90 \%$ Confidence Intervals on Reduction in Expected Relative Delay for Static and Dynamic Stochastic AGV Routing Strategies with No Pedestrians 


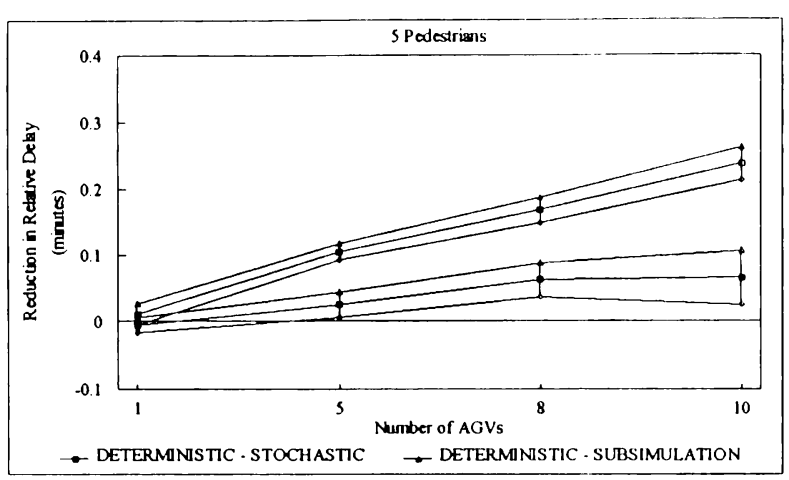

Figure 3: $90 \%$ Confidence Intervals on Reduction in Expected Relative Delay for Static and Dynamic Stochastic AGV Routing Strategies with 5 Pedestrians

\section{ACKNOWLEDGMENTS}

The work reported in this paper was supported by the Fulbright Commission, Germany.

\section{REFERENCES}

Andreatta, G. 1987. Shortest path models in stochastic networks. In Stochastics in combinatorial optimization, ed. G. Andreatta, F. Mason, and P. Serafini. Singapore: World Scientific Publishing Co.

Co, C. G., and J. M. A. Tanchoco. 1991. A review of research on AGVS vehicle management. Engineering Costs and Production Economics 21:35-42.

Hodgson, T. J., R. E. King, S. K. Monteith, and S. R. Schultz. 1987. Developing control rules for an AGVS using Markov decision processes. Material Flow 4:85-96.

Kay, M. G. 1992. Global vision for the control of freeranging automatic guided vehicle systems. Ph.D. dissertation, Department of Industrial Engineering, North Carolina State University, Raleigh, North Carolina.

Kernighan, B. W., and D. M. Ritchie. 1988. The C Programming Language. 2d ed. Englewood Cliffs, New Jersey: Prentice Hall.

Law, A. M., and W. D. Kelton. 1991. Simulation modeling and analysis. 2d ed. New York: McGraw-Hill, Inc.

Pritsker, A. A. B. 1974. The GASP IV simulation language. New York: John Wiley \& Sons.

Pritsker, A. A. B. 1995. Introduction to simulation and SLAM II. 4th ed. New York: John Wiley \& Sons.
Seifert. R. W. 1994. Evaluation of routing strategies for AGVs based on computer simulation. Master's thesis, Integrated Manufacturing Systems Engineering Institute, North Carolina State University, Raleigh, North Carolina.

Viswanadham N., and Y. Narahari. 1992. Performance modeling of automated manufacturing systems. Engelwood Cliffs, New Jersey: Prentice Hall.

\section{AUTHOR BIOGRAPHIES}

RALF W. SEIFERT is a graduate student in the Department of Mechanical Engineering at the University of Karlsruhe (TH) in Germany. His current research interests include manufacturing operations management and simulation aided management. In 1992 he completed a Vordiplom in Mechanical Engineering at the University of Karlsruhe (TH), and he received the Grashof Prize as well as the Prize of the Jubiläumsstaatsstiftung of the University of Karlsruhe (TH). In 1994 he received the degree of Master of Integrated Manufacturing Systems Engineering from North Carolina State University, U.S.A. He is a scholar of the Fulbright Commission in Germany, a scholar of the National German Scholarship Foundation, and a member of the Siemens International Student Circle.

MICHAEL G. KAY is an Assistant Professor in the Department of Industrial Engineering at North Carolina State University. His current research interests are focused in sensor-based control of free-ranging AGV systems. He also has research interests in the areas of material handling, facilities layout and location, real-time control, and multisensor integration and fusion. He received the Ph.D. degree in industrial engineering from North Carolina State University. He has served as Program Cochair of the 1994 IEEE International Conference on Multisensor Fusion and Integration and is a member of IEEE, IIE, and INFORMS.

JAMES R. WILSON is Professor and Director of Graduate Programs in the Department of Industrial Engineering at North Carolina State University. His current research interests are focused on the design and analysis of simulation experiments. He also has an active interest in applications of operations research techniques to all areas of industrial engineering. From 1988 to 1992, he served as Departmental Editor of Management Science for Simulation. He was Proceedings Editor for WSC '86, Associate Program Chair for WSC ' 91 , and Program Chair for WSC ' 92 . He is a member of ASA, ACM/SIGSIM, IIE, and INFORMS. 